\title{
Towards Real-Time Heavy Goods Vehicle Driving Behaviour Classification in the United Kingdom
}

\author{
Utkarsh Agrawal $^{1, *}$, Jimiama Mafeni Mase ${ }^{1, *}$, Grazziela P. Figueredo ${ }^{1,2}$, \\ Christian Wagner ${ }^{1}$, Mohammad Mesgarpour ${ }^{3}$, Robert I. John ${ }^{1}$ \\ ${ }^{1}$ School of Computer Science, The University of Nottingham \\ ${ }^{2}$ The Advanced Data Analysis Centre, The University of Nottingham \\ ${ }^{3}$ Microlise, Farrington Way, Eastwood, Nottingham
}

\begin{abstract}
1 Abstract-Determining the driving styles and the factors causing incidents in real time could assist stakeholders to promote actions and develop feedback systems to reduce risks, costs and to increase safety in roads. This paper presents a classification system for Heavy Goods Vehicles (HGVs) drivers, using a core set of driving pattern stereotypes which were uncovered from driving incidents across three years i.e. 2014, 2015 and 2016. To achieve that, the driving stereotypes are established by employing a 2-stage ensemble classification framework followed by a profile labelling algorithm to define the set of driving stereotypes. Very similar stereotypes are later merged to form the core driving stereotypes for UK HGV drivers. Upon establishing core driving stereotypes across these three years, a decision tree classifier learns the classification rules to identify the driving stereotypes for the HGV drivers in a new dataset. High accuracy is achieved, indicating that the core driving patterns uncovered in this work could potentially be employed to identify UK HGV driving patterns in real-time.
\end{abstract}

Index Terms-Clustering Analysis, Driver Behaviour, Driving Pattern, Driving Stereotypes, Driver Profiling, Ensemble Classification, Ensemble Clustering

\section{INTRODUCTION}

Advances in ubiquitous computing, wireless communication and the widespread use of sensors in transportation has allowed for advances in vehicle health monitoring [1], [2], general transport maintenance [3]-[7] and incident prevention conditioned to data gathered regarding human behaviour [8][10]. Research in driving behaviour, vehicle monitoring, driving style prediction and driver modelling has therefore been increasing significantly over the last decade, as there is high demand for intelligent solutions to improve driving economy and safety. To achieve this, the understanding and prediction of a driver's behaviour and their responses to factors such as road obstacles, different weather conditions and vehicle characteristics is necessary.

In particular, the interest in this work lies in Heavy Goods Vehicle (HGV) driving behaviour, as HGV's transportation is needed for trading of goods by almost every sector of business in the United Kingdom (UK). In this paper, we aim to identify core driving profiles and create a classification system for drivers based on their behaviour extracted from datasets of incidents collected via telematics. We believe the characterisation and classification of HGV driving styles will

\footnotetext{
$1, *$ Both the authors have equally contributed to the work
}

assist transportation research and industry to establish groupspecific plans of action to improve driving economy and safety. In addition, the core classes could be employed to assist defining industry standards in the UK HGV community.

For the analysis in this paper, the data is gathered and provided by Microlise [11], our industrial partner. Microlise employs telematics solutions for driving and vehicle data collection; and they use this information to define strategies for incident prevention and to promote better practices in the HGV industry. In a recent research by Figueredo et al. [12], the authors determine drivers' behavioural stereotypes using the telematics data of drivers from the year of 2015. From their analysis, eight clusters of behaviour are identified. Their study comes from the necessity to better understand how HGV drivers in the UK behave so that Microlise could develop better safety policies. In addition, the work was also motivated by the scarcity of related literature on HGVs.

This paper aims to create a UK HGV drivers classification system. In order to achieve this, two main research objectives are identified: (1) To establish a core set of driving profiles using driving incidents from three years i.e. 2014, 2015 and 2016. By defining these core classes, a more general and robust classification of drivers can be achieved. (2) To establish a classification system to identify driving stereotypes of new drivers from year 2017. Subsequently, validate the classification of these new drivers.

To achieve the first objective, a 2-Stage Classification framework involving consensus clustering and consensus classification is employed, similarly to Figueredo et al. [12]. Eleven clusters (classes) were identified from three telematics datasets from the years 2014, 2015 and 2016. To determine the core driving profiles, some of these eleven clusters with high similarities are merged, resulting in a total of seven core clusters (explained in detail in Section IV-A). As a result, robust core driving stereotypes are obtained, which are present across multiple years. For the second objective, first decision trees are trained to learn these core driving stereotypes. Subsequently, these decision trees are used to classify new set of drivers from the year 2017. Results show high accuracy in the classification system. The classification results are also validated by repeating the 2-stage classification framework to the 2017 data. This validation is done to cross match the decision tree classification results. 
This work is organised as follows, in Section II we review the literature and provide the necessary background to understand the data set and our methodology. This section therefore introduces the 2-stage classification framework and the profile labelling algorithm employed to establish the core driving profiles. In Section III, the dataset description, the algorithm settings, and the steps employed to achieve the two aims are introduced. In Section IV the results are presented along with discussion. Section V concludes this paper and establishes the opportunity for future work.

\section{BACKGROUND}

\section{A. Related Work}

An important contribution to understanding driving behaviour from telematics data is found in Constantinescu et al. [13]. The authors identify six risky driving stereotypes in a controlled experiment, using the Hierarchical Clustering Algorithm. 200 journeys for 25 drivers in the city of Bucharest having four driving features were analysed i.e. speed, acceleration, braking and engine kinetic energy. Similarly, Ellison et al. [14] propose a driver risky index framework based on telematics data i.e. over speed, harsh acceleration and harsh braking, collected from 106 drivers in the city of Sidney within 25 days of driving.

Saiprasert et al. [15] calculate a driver's safety index based on their driving events and categorise the driver into very safe, safe, aggressive, and very aggressive. Their calculation is based on five driving events i.e. harsh acceleration, harsh braking, harsh turning, sudden lane changing and over speed events, depending on road characteristics. The study analyses data from 20 drivers in two of Thailands main highways, with 30 journeys per route. Similarly, Kalsoom and Halim [16] identify three driving profiles from a dataset containing 70 journeys for 30 drivers. Nine driving features are analysed using K-Means and Hierarchical Clustering Algorithms.

Halim et al. [17] also employs clustering algorithms i.e. KMeans, fuzzy c-means and Model-Based Clustering (MBC), to determine four driving profiles from 50 drivers, using 12 driving features in a controlled environment. Each subject drives the car within high, average and low traffic scenarios for 15 minutes each. The results of the clustering methods are analysed to establish the optimal number of driving profiles.

The research reviewed above employs different variations of clustering algorithms to identify groups of driving stereotypes. Their limitations regard mostly the number of samples analysed, which is relatively small, and their experiments are performed in controlled environments, which is unrepresentative of real driving behaviours. In addition, the authors perform no further tests on the validity of the clusters identified.

In Figueredo et. al [12], a more robust model involving a combination of ensemble clustering and ensemble classification is employed to a much larger data set consisting of driving incidents of 21,193 drivers, collected over the year of 2015. Further details on their methodology are presented in the next section. Eight driving stereotypes amongst the UK HGV driving community are identified within three subgroups of short, medium and long average daily mileage. Their work is the first step towards creating a universal set of UK driving stereotypes, with the aim of using the identified stereotypes as industry standards for safe driving in the UK HGV community. In this work, we extend the research from Figueredo et. al [12] by creating and validating a real-time driving pattern identification system. We also identify core driving profiles which are present across the years investigated, with the aim to assist establishing industrial standards.

\section{B. Dataset Description}

The dataset utilised in this study is provided by Microlise [11]. Information produced by their telematics frameworks are transmitted and collected from the HGVs in real time. Data is captured by sensors connected to multiple electronic control units using a Controller Area Network (CAN) bus. The information is transferred to a telematics unit that populates Microlise's databases via $3 \mathrm{G}$. The HGV drivers must complete a minimum of 10 journeys per quarter of each year to be incorporated in the dataset investigated. Every driver must have therefore travelled at least 40 journeys yearly on any road in the UK to be considered in our analysis. The data was collected between the first of January and the thirty first of December for the years 2014, 2015, 2016, and 2017. In total, 15,893 drivers for 2014, 21,234 drivers for 2015, 34,675 drivers for 2016, and 35,432 drivers for 2017 were collected using the criteria mentioned above. Table I shows the distribution of the drivers for the four years within the three driving subgroups i.e., short, medium and long average daily mileage subgroups. Drivers in the year 2014-2016 were used to establish core driving profiles. The drivers in the year 2017 will be classified using a classification model trained on these core profiles.

TABLE I: Distribution of drivers among Average Daily Mileage groups for years 2014, 2015, 2016 and 2017

\begin{tabular}{|c|c|c|c|c|}
\hline $\begin{array}{c}\text { Average daily } \\
\text { mileage groups }\end{array}$ & $\begin{array}{c}\text { Distribution of } \\
\text { drivers in 2014 }\end{array}$ & $\begin{array}{c}\text { Distribution of } \\
\text { drivers in 2015 }\end{array}$ & $\begin{array}{c}\text { Distribution of } \\
\text { drivers in 2016 }\end{array}$ & $\begin{array}{c}\text { Distribution of } \\
\text { drivers in 2017 }\end{array}$ \\
\hline Short & 3,327 & 5,076 & 16,281 & 10,745 \\
\hline Medium & 6,419 & 8,392 & 10,232 & 14,833 \\
\hline Long & 6,147 & 7,766 & 8,162 & 9.854 \\
\hline Total & 15,893 & 21,234 & 34,675 & 35,432 \\
\hline
\end{tabular}

\section{The 2-Stage Classification Framework}

To establish the driving profiles a 2-stage framework was introduced in [12], which incorporated a consensus clustering followed by ensemble classification to cluster the data set as shown in Figure 1. In the first stage, the data is normalised and pre-processed to remove any inconsistencies and noise. Subsequently, multiple clustering algorithms are employed and then aligned to achieve a consensus [18] as to which group each instance belongs to. In the second stage, ensemble classification (majority voting) is used to classify the unclustered data left behind by the ensemble clustering algorithms in the previous stage [19]. Together they form a 2-stage classification framework, as further detailed below.

\section{Stage1: Ensemble Clustering}


As the number of clusters are unknown, to determine their optimal number, validity indices was used. According to specific rules, these validity indices indicate the appropriate number of groups to be considered. K-Means [18] and Partitioning Around Medoids (PAM) [20] algorithms were employed to cluster the data. Subsequently, the consensus among the clustering techniques was determined for each data instance. After employing the consensus clustering, the instances which have no consensus (i.e. one common cluster to which they belong), were labelled as unclassified. After this stage, the main driving behaviour groups are uncovered.

\section{Stage 2: Ensemble Classification}

The objective of this second stage is to tackle the unclassified data from previous stage. The data clustered in Stage 1 will now be employed as training set (for classification algorithms to learn) and the unclassified instances will be used as the test set. The objective was to associate the unclassified data with their closest cluster or to potentially identify a new cluster, which was not detected in Stage 1. Majority voting ensemble classification using Support Vector Machine (SVM), Nearest Neighbour(NN) and Multi-layer Perceptron (MLP) models was employed to learn the patterns. Subsequently, the new clusters were compared with the previously identified clusters after Stage 1 using the Mann-Whitney-Wilcoxon nonparametric t-test (at a 0.05 significance level). If the new clusters were similar to any of the previous clusters, the drivers in the new cluster were combined with the drivers previously clustered after Stage 1. Otherwise, the new cluster was checked for the possibility of being an extra profile. The final classification was achieved by combining results from both stages.

\section{Driving Profile Labelling Algorithm}

To establish driver profiles (driving stereotypes), a labelling algorithm was employed, as defined in [12]. It first removes possible outliers to avoid any drastic shifts on the median values for the data set attributes. Subsequently, it compares the median for each variable of each cluster (i.e., harsh braking, over speed, excessive throttle and over revs) with the quartiles in the box-plot for the entire group data. The categories defined for the attributes were low, moderate, high and very high occurrence of incidents. For each incident variable, if the median of the variable is less or equal to the median of all data in the cluster for that variable, the corresponding label is "low". If the median is between the median and the third quartile, the label is "moderate". If the median is between the third quartile and the maximum value then the label should be "high". Any values in the box plot above the maximum (outliers) should be labelled "very high" (the pseudocode for the profile labelling algorithm is found in the Appendix).

After reproducing the work reviewed in this section, we employ the clusters identified to label the data sets from 2014 2016 and subsequently use it to train our classifier models. In the next section, we provide further details of the data sets used, and introduce the classification of the data, which led to further characterisation of the core HGV driving profiles.

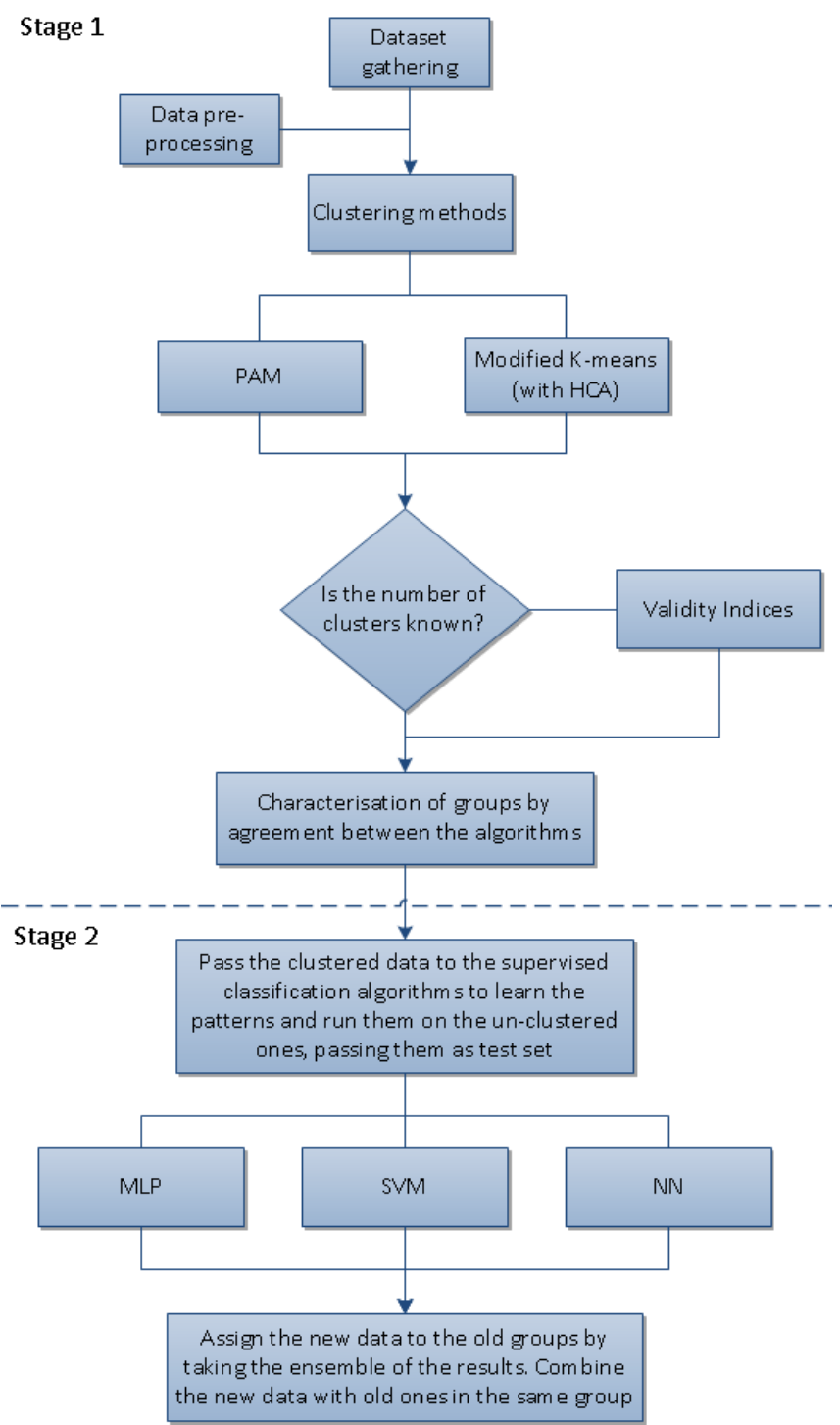

Fig. 1: Flowchart of the pipeline to determine the clusters in the drivers data set (adapted from [12])

\section{Methodology}

\section{A. Data Pre-processing}

For unbiased results, the analysis in this paper is performed by normalising the driving features. Therefore, the number of harsh braking events, over-speed duration in seconds, excessive throttle duration in seconds, and number of over rev events is normalised by dividing with the total driving time in seconds. The average distance driven per day is adapted instead of total distance, as it seems better suited to detect driving behaviour. Figueredo et al [12] uncovered three clusters based on the daily average mileage travelled i.e. short, medium and long daily average mileage. Drivers in the short subgroup cover an average daily mileage between 22.37 and 136.70 miles, drivers in the medium subgroup cover an average daily mileage between 136.70 and 217.48 miles, while drivers in the long subgroup cover between 217.48 and 466.03 miles. 
In this paper, we use these findings to group drivers among these three subgroups.

In this study, we aim to establish core driving stereotypes, therefore it is important to have drivers who are consistently present across all the years. As a result, the study only considers drivers present in all four years i.e. 2014, 2015, 2016, and 2017 , who did not change subgroups across the years i.e. short, medium and long average daily mileage subgroups. For example, if a driver is considered for our study as a short average daily mileage driver in 2014 , they should also travel as a short average daily mileage driver in the years 2015, 2016 and 2017. A total of 2,462 drivers which are present across all the four years is therefore considered in this new analysis.

\section{B. Experiment Steps}

There are two objectives defined and the steps for their respective experiments are described as follows:

1) Identification of core driving patterns:

Step 1 Sub-groups division: Divide the 2,462 drivers from the years 2014, 2015 and 2016 into short, medium and long daily average mileage drivers. This step results in 569, 964 and 929 drivers across short, medium and long average daily mileage subgroups respectively.

Step 2 Pre-processing: Normalise the four driving features i.e. harsh braking events, over-speed duration in seconds, excessive throttle duration in seconds, and number of over rev events by dividing with total driving time (in seconds).

Step 3 Pattern identification: Run the 2-stage classification framework described in Section II-C on each year's dataset for all the three sub-groups separately. Upon uncovering the driving groups, distribute the drivers in one of them.

Step 4 Driving stereotypes across years 2014, 2015, and 2016: Run the profile labelling algorithm described in Section II-D for all the three sub-groups independently in each year to label the groups obtained in Step 3.

Step 5 Identifying core driving stereotypes: Compare the driving patterns observed in the previous step and subsequently merge the similar patterns to form core driving stereotypes.

2) Classification of new drivers:

Step 6 Train decision trees for subgroup classification: To learn the classification rules for identifying UK HGV driving stereotypes in the year 2017, train the three Decision Trees for the three driving groups using the core driving stereotypes established in step 5.

Step 7 Validation of classification system: To validate the classification results observed in step6, compare these classification results with driving patterns obtained by running the 2-stage framework on the drivers in year 2017.

\section{Experimental Settings}

Stage 2 of the classification framework employees an ensemble classification framework with SVM, Nearest Neigh- bour (NN) and Multi Layer Perceptron (MLP) as base classifiers. SVM model with a linear kernel and a penalty parameter of 100, NN model with 8 neighbours and MLP model with 1 hidden layer made up of 130 neurons, Relu activation function $\&$ Adam optimisation, are used. These hyper-parameters are obtained by using a random search optimisation method [21] which is efficient for huge datasets with large parameter sets.

To learn the classification rules of the core driving stereotypes, we use C4.5 algorithm [22] to generate three binary Decision Tree (DT) models for short, medium and long average daily mileage drivers. The three models used entropy information gain with 10-fold cross-validation and 3, 3 and 7 tree depths respectively. The hyper-parameters are obtained by using random search optimisation method.

\section{RESUlts AND Discussion}

For consistency, this study considers only drivers present in all the years who did not change subgroups. A total of 2,462 drivers are considered, i.e. 569, 964 and 929 drivers for short, medium and long average daily mileage subgroups, respectively. Table II shows 11 profiles uncovered after running the 2 stage ensemble clustering - ensemble classification framework and the profile labelling algorithm on each subgroup for 2014, 2015 and 2016 datasets. The first column defines the profile number, columns two to five define the category of the number of incidents for the four driving features in each profile obtained using the labelling algorithm. The last three columns indicate the year and subgroups where the profiles are present. S, M and L stand for short, medium and long average daily mileage subgroups respectively.

It can be observed that profile 1 is present across all three years, having safe drivers with low incidents for all the driving features except for number of over revs which has a moderate number of incidents. Profiles 2 and 3 drivers have similar harsh braking, over speed and excessive throttle incidents but differ in the number of over revs. Profiles 4,5 and 6 are again very similar in all the driving features except for number of over revs. Similarly, profiles 7 and 8 differ only in excessive throttle incidents i.e. moderate for profile 7 and high for profile 8 . Profiles 9 and 10 differ in over-speed duration i.e. moderate over-speed duration for profile 9 and high over-speed duration for profile 10. Overall, profiles 8 and 10 represent aggressive drivers with atleast two driving features with high incident numbers. Moreover, it is interesting to observe that profile 10 is present across all the subgroups for the three years (except short subgroup in year 2014), indicating that every year there are drivers with aggressive behaviour. Lastly, profile 11 represents very aggressive drivers with three high driving features.

When analysing longitudinally i.e. changes across the three years, it can be observed that driving behaviour is consistent. For example, the years 2014 and 2015 have four common driving profiles i.e. profiles $1,4,10$ and 11. Moreover, profile 2 for 2014 and profile 3 for 2015 are also similar as they differ only in the number of over rev incidents (low over revs for profile 2 and moderate over revs for profile 3). Similarly, 
TABLE II: HGV driving profiles uncovered across 2014, 2015, and 2016

\begin{tabular}{|c|c|c|c|c|c|c|c|}
\hline $\begin{array}{c}\text { Profile } \\
\text { Number }\end{array}$ & $\begin{array}{c}\text { Harsh } \\
\text { Braking }\end{array}$ & $\begin{array}{c}\text { Over-Speed } \\
\text { duration }\end{array}$ & $\begin{array}{c}\text { Excessive } \\
\text { Throttle }\end{array}$ & $\begin{array}{c}\text { Number of } \\
\text { Over Revs }\end{array}$ & 2014 & 2015 & 2016 \\
\hline 1 & Low & Low & Low & Moderate & S,M,L & S,M,L & S,M,L \\
2 & Low & Low & High & Low \\
3 & Low & Low & High & Moderate & - & S & S \\
4 & Moderate & Moderate & High & Low & L & L & - \\
5 & Moderate & Moderate & High & Moderate & S,M & - & L \\
6 & Moderate & Moderate & High & High & - & - & M \\
7 & Moderate & High & Moderate & Low & S & - & - \\
8 & Moderate & High & High & Low & L & - & - \\
9 & High & Moderate & Moderate & Low & M & - & - \\
10 & High & High & Moderate & Low & M,L & S,M,L & S,M,L \\
11 & High & High & High & Low & M & L & - \\
\hline
\end{tabular}

TABLE III: Core Driving Profiles

\begin{tabular}{|c|c|c|c|c|c|c|c|}
\hline $\begin{array}{l}\text { Profile } \\
\text { Number }\end{array}$ & $\begin{array}{c}\text { N. of Harsh } \\
\text { Braking Events }\end{array}$ & $\begin{array}{c}\text { N. of Over } \\
\text {-speed Events }\end{array}$ & $\begin{array}{l}\text { Excessive } \\
\text { Throttle }\end{array}$ & $\begin{array}{l}\text { N. of Over } \\
\text { Revs Events }\end{array}$ & $\begin{array}{c}2014 \\
\text { Sub-groups }\end{array}$ & $\begin{array}{c}2015 \\
\text { Sub-groups }\end{array}$ & $\begin{array}{c}2016 \\
\text { Sub-groups }\end{array}$ \\
\hline 1 & Low & Low & Low & Moderate & S,M,L & S,M,L & S,M,L \\
\hline 2,3 & Low & Low & High & Low-Moderate & $\mathrm{M}$ & $\mathrm{S}$ & $\mathrm{S}$ \\
\hline 4,5 & Moderate & Moderate & High & Low-Moderate & S,M,L & $\mathrm{L}$ & $\mathrm{L}$ \\
\hline 6 & Moderate & Moderate & High & High & - & - & $\mathrm{M}$ \\
\hline 7,8 & Moderate & High & Moderate-High & Low & S,L & - & - \\
\hline 9,10 & High & Moderate-High & Moderate & Low & M,L & S,M,L & S,M,L \\
\hline 11 & High & High & High & Low & $\mathrm{M}$ & $\mathrm{L}$ & - \\
\hline
\end{tabular}

years 2015 and 2016 have three common driving profiles i.e. profiles 1, 3, and 10. Profile 4 for 2015 and profile 5 for 2016 only differ in the number of over rev incidents (low over revs for profile 4 and moderate over revs for profile 5).

\section{A. UK HGV Core Driving Profiles}

To produce the core driving profiles for UK HGV drivers, driving profiles which have similar patters are merged. Profiles which differ by only one driving category (i.e. low or moderate or high or very high) in only one of the driving attributes (i.e. harsh braking or Over-speeding or Excessive Throttle or Over revs) are also merged. For example, profiles 2 and 3 differ only in number of Over revs, one being low and the other being moderate; they can therefore be merged to form one stereotype. Similarly, profiles 4,7 and 9 are combined with profiles numbers 5, 8 and 10, respectively. Profiles 1,6 and 11 are standalone stereotypes as they do not follow the aforementioned rule.

Table III shows the seven core driving stereotypes deduced from the 11 profiles uncovered across the three years and Table IV shows the distribution of drivers in these core driving stereotypes from 2014-2017. The first core driving stereotype is formed by profile 1 and represents a safe driving stereotype with low driving features except number of over revs events. This profile also has the highest number of drivers across the years. Two aggressive stereotypes are produced by profiles 7,8 and 9,10 respectively, with both stereotypes found in 2014. One very aggressive stereotype is established by profile 11 which is present in both 2014 and 2015. It is interesting when analysing these core profiles longitudinally, 2014 has 2 aggressive and 1 very aggressive profiles, 2015 has 1 aggressive and 1 very aggressive profiles, while 2016 only has 1 aggressive profile. This observation clearly shows an improvement in an overall $\mathrm{HGV}$ driving performance across these three years.

\section{B. Classification of New $\mathrm{HGV}$ drivers}

Three DT classifiers are trained for short, medium and long average daily mileage subgroups using the seven core driving patterns uncovered previously (Table III). For year 2017, a driver with a short average daily mileage will be classified using the short average daily mileage DT, similarly, a driver with a medium average daily mileage will be classified using the medium average daily mileage DT and lastly, a driver with a long average daily mileage will be classified using the long average daily mileage DT. Figure 2 shows the classification rules for short daily average mileage drivers (the decision trees for the medium and long daily average mileage drivers are found in the Appendix). It can be observed that the over-speed driving feature is the root node of the decision trees, indicating this is the most significant driving feature in classifying $\mathrm{HGV}$ drivers in real time. The second most important feature being excessive throttle, followed by harsh braking and over revs driving features. Similar order was observed in the other two classification models.

To validate the classification of new drivers (i.e. the drivers in year 2017), these results are compared with the profiles obtained by running the 2-stage classification framework on the drivers from year 2017. For short average daily mileage drivers, $97.85 \%$ of the drivers match between classification using DT with those uncovered by the 2-stage framework. Similarly, for medium and long average daily mileage drivers, $80.68 \%$, and $87.13 \%$ match respectively. The results show 


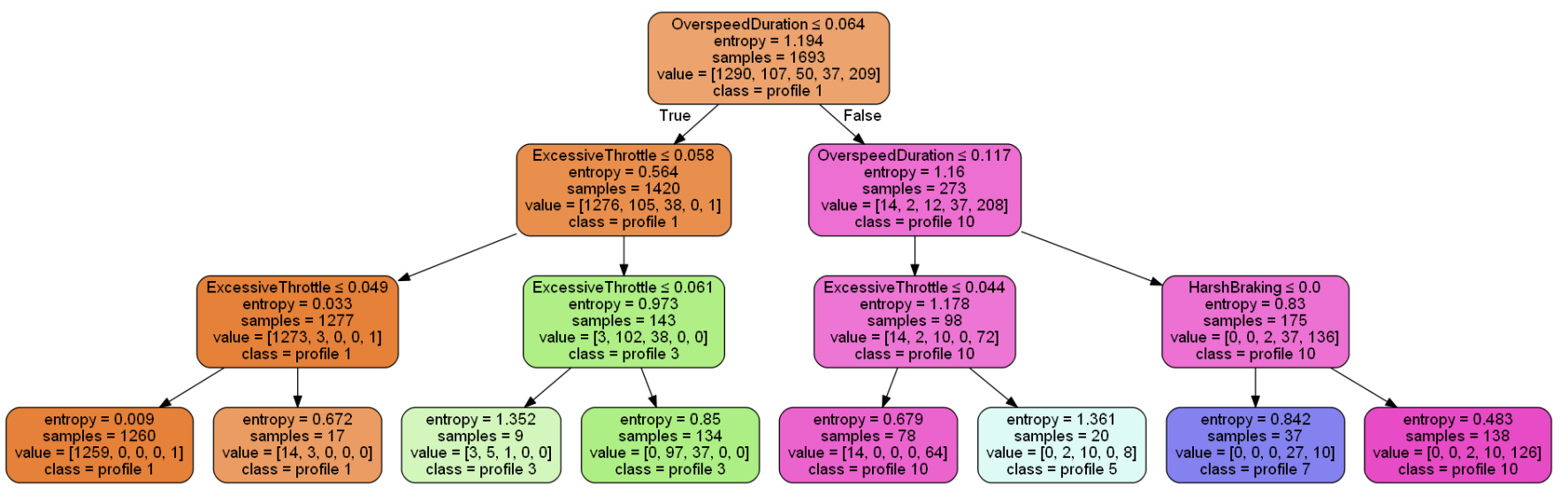

Fig. 2: Decision Tree Classification Rules for short daily average distance drivers

that the classification system can be used to identify driving stereotypes in real-time and with high confidence.

TABLE IV: Distribution of drivers in Core Driving Profiles

\begin{tabular}{|c|c|c|c|c|}
\hline $\begin{array}{c}\text { Profile } \\
\text { Number }\end{array}$ & $\begin{array}{c}\text { Distribution o f } \\
\text { drivers in 2014 }\end{array}$ & $\begin{array}{c}\text { Distribution of } \\
\text { drivers in 2015 }\end{array}$ & $\begin{array}{c}\text { Distribution of } \\
\text { drivers in 2016 }\end{array}$ & $\begin{array}{c}\text { Distribution of } \\
\text { drivers in 20117 }\end{array}$ \\
\hline 1 & 1,792 & 1,621 & 1,617 & 1,650 \\
\hline 2,3 & 43 & 55 & 52 & 66 \\
\hline 4,5 & 234 & 81 & 59 & 64 \\
\hline 6 & - & - & 66 & - \\
\hline 7,8 & 86 & - & - & - \\
\hline 9,10 & 194 & 591 & 612 & 640 \\
\hline 11 & 52 & 70 & - & - \\
\hline
\end{tabular}

\section{CONCLUSion AND Future Work}

In Figueredo et al. [12], the authors employed a 2-Stage framework to identify eight driving profiles within a telematics dataset containing the driving incidents of the HGV drivers. This study builds upon the aforementioned work to achieve two objectives: (1) to establish a core set of driving stereotypes representing UK HGV driving behaviour; and to (2) use these core driving stereotypes to construct a robust classification system which can classify the new drivers in real-time.

To achieve the first objective, we employ the 2-stage classification framework on dataset containing the driving incidents across U.K. HGV drivers for the years 2014, 2015 and 2016. For unbiased results, the analysis was performed by dividing the drivers among the short, medium and long average daily distance travelled. A total of eleven driving profiles were uncovered among the drivers who were present across all these years for all the three categories (i.e. short, medium and long average daily distance) combined. To establish the core driving stereotypes, very similar profiles were merged, resulting in a total of seven core driving stereotypes of UK drivers.

For the second objective, three decision trees were trained to learn the classification rules of the core driving stereotypes across short, medium and long average daily distance drivers respectively. New drivers from the year 2017 were classified among one of the driving profiles using these decision trees. The classification performance was validated by comparing their results with those obtained from employing the 2-stage framework on the year 2017. For short average daily mileage drivers, $97.85 \%$ of the drivers classified using decision trees matched those uncovered by the 2-stage framework. Similarly, for medium and long average daily mileage drivers, $80.68 \%$, and $87.13 \%$ match respectively. The results show that the classification system can be used to identify driving stereotypes in real-time with high confidence. In addition, operators can develop strategies and systems that give feedback to the drivers in real time to improve their driving habits. Examples of those strategies range from making helpful recommendations, creating interactive games, sending warning messages, to directly interfering and taking the lead on driving, so as to optimise safety without degrading the joy experience of driving.

Lastly, some limitations and scope for future works are discussed.

Limited number of features: To identify driving stereotypes, this study only analysed four driving features i.e. harsh braking events, over-speed duration in seconds, excessive throttle duration in seconds, and number of over rev events. For future works, we plan to include more driving features such as harsh cornering, lane changing and close following, which seem to be relevant for HGV and cargo safety and might be significant in improving the stereotype classification accuracy.

Uncertainty in captured data: While capturing the data in the real world, a number of uncertainties might be injected in the sensor readings. Moreover, the combination of different driving features and subjective decisions made by experts could lead to different results. For example, a driving feature considered 'low' by one expert may be regarded moderate by another expert. As a result, we plan to upgrade our system and analyse the captured data using the fuzzy sets in order to better handle the uncertainty, imprecision and subjectivity.

Other facets of driver behaviour: Our study only explores the use of telematics data for detecting driver behaviour but lacks the analysis of other facets of driver behaviour that also affect driving performance such as driver distraction, workload, situation awareness, stress, fatigue and attention. These psychological and human factors of drivers can be captured using several technologies such as cameras and Elec- 
troencephalography (EEG) signals. Combining these different measures of driver behaviour can improve the accuracy in detecting risky behaviours or provide a more reliable holistic view of driver behaviour to assist stakeholders in the decision making process.

External factors: Driving incidents are influenced by several external factors such as weather conditions, traffic conditions, time of the day, company policies, and in-vehicle technologies. In order to determine incidents caused by drivers, we need to filter out the external factors that affect driving performance. Therefore, we plan on exploring the effects of these factors on driver performance using big data in a naturalistic driving environment.

Real time classification: The main objective of our classification system is to detect driving stereotypes in real time. This classification system can enable operators to monitor safety risk and develop interventions to alleviate potential problems and prevent crashes in real time. Therefore, in future we aim to construct a classification model which can identify driving stereotypes in real time using the incoming data streams and by examining aforementioned drawbacks and including the proposed updates.

\section{ACKNOWLEDGEMENTS}

The authors would like to thank Microlise for providing us the data.

\section{REFERENCES}

[1] M. Mesgarpour, D. Landa-Silva, and I. Dickinson. Overview of telematics-based prognostics and health management systems for commercial vehicles. Activities of Transport Telematics, 395:123-130, 2013.

[2] M. Mesgarpour and I. Dickinson. Enhancing the value of commercial vehicle telematics data through analytics and optimisation techniques. Archives of Transport System Telematics, 7(3):27-30, 2014.

[3] M Marseguerra, E Zio, and L Podofillini. Condition-based maintenance optimization by means of genetic algorithms and Monte Carlo simulation. Reliability Engineering \& System Safety, 77(2):151-165, 2002.

[4] E Safari and Seyed J Sadjadi. A hybrid method for flowshops scheduling with condition-based maintenance constraint and machines breakdown. Expert Systems with Applications, 38(3):2020-2029, 2011.

[5] Minou C.A. Olde Keizer, Simme Douwe P. Flapper, and Ruud H. Teunter. Condition-based maintenance policies for systems with multiple dependent components: A review. European Journal of Operational Research, 261(2):405-420, 2017.

[6] Samir Khan and Takehisa Yairi. A review on the application of deep learning in system health management. Mechanical Systems and Signal Processing, 107:241-265, 2018.

[7] D. Rengasamy, H. Morvan, and G. P. Figueredo. Deep learning approaches to aircraft maintenance, repair and overhaul: A review. In submitted to: The IEEE International Conference on Intelligent Transportation Systems, page tbc, Nov 2018.

[8] J. D. Davey, J. E. Freeman, D. E. Wishart, and B. D. Rowland. Developing and implementing fleet safety interventions to reduce harm: Where to from here? In International Symposium on Safety Science and Technology, Beijing, China, 2008.
[9] J. R.D. Edwards, J. Davey, and K. A. Armstrong. Profiling contextual factors which influence safety in heavy vehicle industries. Accident Analysis \& Prevention, 73:340-350, 2014.

[10] G. P. Figueredo, P. R. Quinlan, M. Mesgarpour, J. M. Garibaldi, and R. I. John. A data analysis framework to rank hgv drivers. In 2015 IEEE 18th International Conference on Intelligent Transportation Systems, pages 2001-2006. IEEE, 2015.

[11] Microlise. Microlise (telematics, transport and fleet management solutions). Available at: http://www.microlise.com/, Last accessed 30th April 2018.

[12] G. P. Figueredo, U. Agrawal, J. M. Mase, M. Mesgarpour, C. Wagner, D. Soria, J. M. Garibaldi, P.O. Siebers, and R. I. John. Identifying heavy goods vehicle driving styles in the united kingdom. IEEE Transactions on Intelligent Transportation Systems (in press), tbc:tbc, 2018.

[13] Zoran Constantinescu, Cristian Marinoiu, and Monica Vladoiu. Driving style analysis using data mining techniques. Int J of Comp Communications \& Control, 5(5):654-663, 2010.

[14] Adrian B Ellison, SP Greaves, and Rhonda Daniels. Profiling drivers risky behaviour towards all road users. In A safe system: expanding the reach: Australasian College of Road Safety national conference, 2012.

[15] C. Saiprasert, S. Thajchayapong, T. Pholprasit, and C. Tanprasert. Driver behaviour profiling using smartphone sensory data in a v2i environment. In 2014 Int Conf on Connected Vehicles and Expo, pages 552-557, 2014.

[16] R. Kalsoom and Z. Halim. Clustering the driving features based on data streams. In Multi Topic Conference (INMIC), 2013 16th International, pages 89-94, Dec 2013.

[17] Zahid Halim, Rizwana Kalsoom, and Abdul Rauf Baig. Profiling drivers based on driver dependent vehicle driving features. Appl Int, 44(3):645$664,2016$.

[18] D. Soria, J. M. Garibaldi, F. Ambrogi, A. R. Green, D. Powe, Rakha E., R. Douglas Macmillan, R. W. Blamey, G. Ball, P. J. G. Lisboa, T A Etchells, P Boracchi, E Biganzoli, and I O Ellis. A methodology to identify consensus classes from clustering algorithms applied to immunohistochemical data from breast cancer patients. Computers in Biology and Medicine, 40(3):318-330, 2010.

[19] U Agrawal, D Soria, and C Wagner. Cancer subtype identification pipeline: A classifusion approach. 2016 IEEE Congress on Evolutionary Computation, CEC 2016, pages 2858-2865, 2016.

[20] L. Kaufman and P. Rousseeuw. Clustering by Means of Medoids. Rep of the Fac of Mathematics and Informatics. 1987.

[21] James Bergstra and Yoshua Bengio. Random search for hyper-parameter optimization. Journal of Machine Learning Research, 13(Feb):281-305, 2012.

[22] Bhumika Gupta, Aditya Rawat, Akshay Jain, Arpit Arora, and Naresh Dhami. Analysis of various decision tree algorithms for classification in data mining. Int J Comput Appl, 8:15-9, 2017.

\section{APPENDIX A \\ SUPPLEMENTARY DOCUMENT}

Due to page limitation, we have provided the profile labelling algorithm and decision tree classification results for medium and long daily average mileage driver in our ResearchGate link ${ }^{2}$.

${ }^{2}$ https://www.researchgate.net/publication/331988747_Towards_Real_ Time_Heavy_Goods_Vehicle_Driving_Behaviour_Classification_A_Case_ Study_for_the_United_Kindgom 